\title{
Diffusion on a Hypersphere: Application to the Wright-Fisher model
}

\author{
Kishiko Maruyama \\ Yoshiaki Itoh
}

The Institute of Statistical Mathematics and The Graduate University for Advanced Studies, 10-3 Midori-cho, Tachikawa, Tokyo 190-8562, Japan

\begin{abstract}
The eigenfunction expansion by Gegenbauer polynomials for the diffusion on a hypersphere is transformed into the diffusion for the Wright-Fisher model with a particular mutation rate. We use the Ito calculus considering stochastic differential equations. The expansion gives a simple interpretation of the Griffiths eigenfunction expansion for the Wright-Fisher model. Our representation is useful to simulate the Wright-Fisher model as well as Brownian motion on a hypersphere.
\end{abstract}

PACS numbers: 02.50.Ey, 87.10.Ca 


\section{Introduction}

Here we show that the diffusion on a hypersphere 3 is transformed into the diffusion for the Wright-Fisher model with a particular mutation rate [7, 8, 9], by using the relation, $x_{i}=y_{i}^{2}$, where $x_{i}$ 's denote the relative abundance of alleles and $y_{i}$ 's denote the position of a particle of the diffusion on a hypersphere. Diffusion on a sphere has been applied to various problems in physics, chemistry, mathematics, etc. [4, 22, 28, 1]. The WrightFisher model is widely used to study the random sampling effect in population genetics [5, 6, 16, 26].

We consider stochastic differential equations in the Ito sense [20]. We represent the Wright-Fisher model for $k$ alleles as well as the diffusion on $(k-1)$-dimensional sphere [17], by using $\left(\begin{array}{l}k \\ 2\end{array}\right)$ independent standard Brownian motion $b_{i j}(t) i>j$ with the skew symmetry $b_{i j}(t)+b_{j i}(t)=0$ [11, 13, 14, 18]. Our representation is useful to simulate the Wright-Fisher model [19, 23]. There are effective simulation methods to simulate a Brownian motion on a hypersphere for example [2]. Our representation is simple and may be useful to simulate the Brownian motion on a hypersphere.

\section{Stochastic differential equation for the Wright-Fisher model}

In the Wright-Fisher model each of the genes of the next generation is obtained by a random choice among the genes of the previous generation and that the whole population changes all at once. In Moran's model [21] it is supposed that there are $2 N$ individuals each formed from $k$ alleles $A_{1}, A_{2}, \ldots, A_{k}$, and that each instant at which the state of the model may change, one individual of the alleles, chosen at random, dies and is replaced by a new individual which is $A_{i}$ with probability $\frac{k_{i}}{2 N}$, where $k_{i}$ is the abundance of the allele $A_{i}$. It is supposed that the probability of any individual "dying" during an interval $(t, t+d t)$ and then being replaced by a new individual is $\lambda d t$. Hence the mean number of such events in unit time is $\lambda$ and the mean length of a generation is $\lambda^{-1}$. The following model of interacting particles [12] is equivalent with the Moran model.

Consider a population of $N$ particles each of which is one of $k$ types, $A_{1}, A_{2}, \ldots, A_{k}$. The types may represent species, alleles, genotypes or other classification. We then consider random interactions between particles, which are assumed to occur at the rate $\lambda d t$ per time interval $(t, t+d t)$ for each particle. If a pair of particles of different types $i$ and $j$ interact, then after the interaction the both particles are the type $i$ with probability $1 / 2$ and the type $j$ with probability $1 / 2$. If the type of the interacting particles are the same, no change occurs.

We can approximate the behavior of our interacting particle system by a stochastic differential equation (11). In it, the relative abundance of type $i$ increases by $c \sqrt{x_{i}(t) x_{j}(t)} d b_{i j}(t)$ and decreases by $c \sqrt{x_{i}(t) x_{j}(t)} d b_{i j}(t)$ with the interaction of the particles of type $j$, where $c=\sqrt{\lambda / 2 N}$. Hence our interacting particle system automatically makes the following equation (11), which has the genetic drift matrix with the elements $c^{2} x_{i}(t)\left(\delta_{i j}-x_{j}(t)\right) d t$ for $i, j=1,2, \ldots, k$ as covariances. 
For $i, j=1,2, \ldots, k$, consider

$$
d x_{i}(t)=\sum_{j=1, i \neq j}^{k} c \sqrt{x_{i}(t) x_{j}(t)} d b_{i j}(t)
$$

with $b_{i j}(t)+b_{j i}(t)=0$, where $b_{i j}(t)(i>j)$ are mutually independent one-dimensional Brownian motion with the mean 0 and the variance $t$ [11, 13, 14, 18]. This representation of the Wright-Fisher model [11] is applied to make computer simulations on models in population genetics [19, 23]. Here we make use of this equation (1) to discuss the exact solutions of the diffusion equations considering the diffusion on a unit sphere.

\section{Diffusion on hypersphere}

The isotropic diffusion on the $(k-1)$-dimensional unit sphere is given by the following stochastic differential equation in the Ito sense.

$$
d y_{i}(t)=\frac{-c^{2}}{8}(k-1) y_{i}(t) d t+\frac{c}{2} \sum_{j=1}^{k} y_{j}(t) d b_{i j}(t)
$$

with $b_{i j}(t)+b_{j i}(t)=0$, for $i=1,2, \ldots, k$, where $b_{i j}(t)(i>j)$ are mutually independent one-dimensional Brownian motion with the mean 0 and the variance $t$. Let $\mathbf{d y}=$ $\left(d y_{1}, \ldots, d y_{k}\right)$. By using the Ito calculus [20], we have for the stochastic differential

$$
d\left(\sum_{i} y_{i}(t)^{2}\right)=0
$$

Hence starting from a point on the unit hypersphere, the trajectory is on the unit hypersphere $\sum_{i} y_{i}(t)^{2}=1$. Consider the dot product for a unit vector $\mathbf{l}=\left(l_{1}, l_{2}, \ldots, l_{k}\right)$,

$$
\mathbf{l} \cdot \mathbf{d} \mathbf{y}=\sum_{i} l_{i}\left[\frac{c}{2} \sum_{j}\left(y_{j}(t) d b_{i j}(t)\right)+\frac{-c^{2}}{8}(k-1) y_{i}(t) d t\right] .
$$

On the tangent hyperplane at $\mathbf{y}=\left(y_{1}, y_{2}, \ldots, y_{k}\right)$ of the hypersphere, for $\mathbf{l}$ which is perpendicular to $\mathbf{y}$, we have

$$
\mathbf{l} \cdot \mathbf{d} \mathbf{y}=\frac{c}{2} \sum_{i} l_{i}\left(\sum_{j} y_{j}(t) d b_{i j}(t)\right)
$$

We have the expectation

$$
E(\mathbf{l} \cdot \mathbf{d} \mathbf{y})=0 .
$$

We have

$$
\operatorname{Var}(\mathbf{l} \cdot \mathbf{d y})=\left(\frac{c}{2}\right)^{2} \sum_{i>j}\left(l_{i} y_{j}-l_{j} y_{i}\right)^{2} d t=\left(\frac{c}{2}\right)^{2} d t
$$

since

$$
\begin{aligned}
& \sum_{i>j}\left(l_{i} y_{j}-y_{i} l_{j}\right)^{2}=\sum_{i>j}\left[\left(l_{i} y_{j}\right)^{2}+\left(y_{i} l_{j}\right)^{2}-2 l_{i} y_{j} y_{i} l_{j}\right]+\left(\sum_{i} l_{i} y_{i}\right)^{2} \\
& =\sum_{i>j}\left[\left(l_{i} y_{j}\right)^{2}+\left(y_{i} l_{j}\right)^{2}\right]+\sum_{i}\left(l_{i} y_{i}\right)^{2}=\left(\sum_{i} l_{i}^{2}\right)\left(\sum_{i} y_{i}^{2}\right)=1 .
\end{aligned}
$$


$\operatorname{Var}(\mathbf{l} \cdot \mathbf{d y})$ does not depend on the direction $\mathbf{l}$ at the tangential point $\mathbf{y}$ of the tangential plane. Hence we see the diffusion is isotropic and we have the Fokker-Planck equation on the hypersphere for the Laplace-Beltrami operator [3] in space $S_{k-1}$,

$$
\frac{\partial}{\partial t} \rho\left(\mathbf{y}, t \mid \mathbf{y}^{\prime}, 0\right)=D \triangle^{S_{k-1}} \rho\left(\mathbf{y}, t \mid \mathbf{y}^{\prime}, 0\right)
$$

where $D=\frac{c^{2}}{8}$ from (77). The operator $\triangle^{S_{k-1}}$ is for $k-1$ spherical coordinates $\theta_{1}, \theta_{2}, \ldots, \theta_{k-1}$ of the unit vector $y=\left(y_{1}, \ldots, y_{k}\right)$ of $S_{k-1}$ defined by the relations

$$
\begin{aligned}
y_{1} & =\sin \theta_{k-1} \ldots \sin \theta_{2} \sin \theta_{1} \\
y_{2} & =\sin \theta_{k-1} \ldots \sin \theta_{2} \cos \theta_{1} \\
& \vdots \\
y_{k-1} & =\sin \theta_{k-1} \cos \theta_{k-2} \\
y_{k} & =\cos \theta_{k-1},
\end{aligned}
$$

where $0 \leq \theta_{1}<2 \pi$ and $0 \leq \theta_{i}<\pi$ for $i \neq 1$. The integration measure in $S_{k-1}$ is defined as $d y=\frac{1}{A_{k-1}} \sin ^{k-1} \theta_{k-1} \ldots \sin \theta_{1} d \theta_{1} \ldots d \theta_{k-1}$, where $A_{k-1}=2 \pi^{k / 2} / \Gamma(k / 2)$ is the surface of the sphere $S_{k-1}$. With this normalization $\int_{S_{k-1}} d y=1$.

The solution [3], the transition probability density for the isotropic diffusion on hypersphere, is obtained by using the definitions and notations of the chapter IX of the book [24]. The Gegenbauer polynomial $C_{L}^{p}$ is defined as the coefficient of $h^{L}$ in the power-series expansion of the function

$$
\left(1-2 t h+h^{2}\right)^{-p}=\sum_{L=0}^{\infty} C_{L}^{p}(t) h^{L} .
$$

The transition probability density from $\mathbf{y}^{\prime}$ (with $n-1$ spherical coordinates $\theta_{1}^{\prime}, \ldots, \theta_{n-1}^{\prime}$ ) at 0 to $\mathbf{y}$ (with $\theta_{1}, \ldots, \theta_{n-1}$ ) at $t>0$ is given [3] by

$$
\begin{aligned}
& \rho\left(\mathbf{y}, t \mid \mathbf{y}^{\prime}, 0\right) \\
& =\frac{1}{A_{k-1}} \sum_{L=0}^{\infty} \frac{2 L+k-2}{k-2} C_{L}^{k / 2-1}\left(\mathbf{y} \cdot \mathbf{y}^{\prime}\right) \exp (-D L(L+k-2) t),
\end{aligned}
$$

for the dot product $\mathbf{y} \cdot \mathbf{y}^{\prime}=\sum_{i} y_{i} y_{i}^{\prime}$, where

$$
C_{L}^{(k / 2-1)}(z)=\sum_{j=0}^{\lfloor L / 2\rfloor}(-1)^{j} \frac{\Gamma(L-j+k / 2-1)}{\Gamma(k / 2-1) j !(L-2 j) !}(2 z)^{L-2 j} .
$$

\section{Solution for the Wright-Fisher model with parent-independent mutation}

Let $A_{1}, \ldots, A_{k}$ denote $k$ allele types in a population. The general neutral alleles model has a probability $u_{i j}$ of a mutation from $A_{i}$ to $A_{j}$. An exact solution is known for the case of the 0 mutation rate case in three dimensions [16. Assuming parent-independent mutation [7], $u_{i j}=u_{j}$. an expansion of the transition probability density [7], from the 
Diffusion on a Hypersphere: Application to the Wright-Fisher model

initial relative abudances $\mathbf{x}^{\prime}=\left(x_{1}^{\prime}, \ldots, x_{k}^{\prime}\right)$ to the relative abundances $\mathbf{x}=\left(x_{1}, \ldots, x_{k}\right)$, is obtained for the following Fokker-Planck equation (18),

$$
\frac{\partial p\left(\mathbf{x}, t \mid \mathbf{x}^{\prime}, 0\right)}{\partial t}=\sum_{i=1}^{k-1} \frac{\partial}{\partial x_{i}}\left(\frac{1}{2} \sum_{i=1}^{k-1} \frac{\partial x_{i}\left(\delta_{i j}-x_{j}\right)}{\partial x_{j}}-\frac{1}{2} M_{i}\right) p\left(\mathbf{x}, t \mid \mathbf{x}^{\prime}, 0\right)
$$

where $M_{i}=\varepsilon_{i}-\mu x_{i}, \mu=\sum_{i=1}^{k} \varepsilon_{i}$. For $\varepsilon_{i}>0, i=1,2, \ldots, k$, the stationary density [27, 25] is given by

$$
\Gamma(\mu) \prod_{i=1}^{k} x_{i}^{\varepsilon_{i}-1} / \Gamma\left(\varepsilon_{i}\right)
$$

The solution of equation (18) is given by an expansion in orthogonal polynomials [7]. For the case $\varepsilon_{i}=\varepsilon, i=1, \ldots, k$, we have the solution [7],

$$
\begin{aligned}
& p\left(\mathbf{x}, t \mid \mathbf{x}^{\prime}, 0\right) \\
= & \Gamma(\mu) \prod_{i=1}^{k} \frac{x_{i}^{\varepsilon-1}}{\Gamma(\varepsilon)}\left(\sum_{n=0}^{\infty} \exp \left\{-\frac{1}{2} n(n-1) t-\frac{1}{2} \mu n t\right\} Q_{n}\left(\mathbf{x}, \mathbf{x}^{\prime}\right)\right)
\end{aligned}
$$

for $x_{j}>0, j=1,2, \ldots, k$, where $Q_{n}$ is given by

$$
Q_{n}\left(\mathbf{x}, \mathbf{x}^{\prime}\right)=(\mu+2 n-1)(n !)^{-1} \sum_{m=0}^{n}(-1)^{n-m}\left(\begin{array}{c}
n \\
m
\end{array}\right)(\mu+m)_{(n-1)} \xi_{m}
$$

with

$$
\xi_{m}=\mu_{(m)} \Gamma(\varepsilon)^{k} \sum_{l_{1}+\ldots+l_{k}=m, 0 \leq l_{j}, j=1, \ldots, k} \frac{m !}{l_{1} ! \ldots, l_{k} !} \Pi_{j=1}^{k} \frac{\left(x_{j} x_{j}^{\prime}\right)^{l_{j}}}{\Gamma\left(l_{j}+\varepsilon\right)},
$$

for the notation $\alpha_{(m)}=\alpha(\alpha+1) \cdots(\alpha+m-1)$. We give the particular cases as,

$$
\begin{aligned}
& Q_{0}\left(\mathbf{x}, \mathbf{x}^{\prime}\right)=1, \\
& Q_{1}\left(\mathbf{x}, \mathbf{x}^{\prime}\right)=(\mu+1)\left(\xi_{1}-1\right), \\
& \left.Q_{2}\left(\mathbf{x}, \mathbf{x}^{\prime}\right)=\frac{1}{2}(\mu+3)\left[(\mu+2) \xi_{2}-2(\mu+1) \xi_{1}+\mu\right)\right],
\end{aligned}
$$

with

$$
\begin{aligned}
& \xi_{1}=\mu \sum_{j=1}^{k} \frac{x_{j} x_{j}^{\prime}}{\varepsilon} \\
& \xi_{2}=\mu(\mu+1) \sum_{j=1}^{k}\left(\frac{\left(x_{j} x_{j}^{\prime}\right)^{2}}{\varepsilon(\varepsilon+1)}+2 \sum_{i<j} \frac{x_{i} x_{j} x_{i}^{\prime} x_{j}^{\prime}}{\varepsilon^{2}}\right) .
\end{aligned}
$$

For $i=1, \ldots, k$, putting $y_{i}^{2}=x_{i}$, the isotropic diffusion (2) on $(k-1)$-dimensional sphere is transformed to

$$
d x_{i}(t)=\frac{c^{2}}{4}\left(1-k x_{i}(t)\right) d t+\sum_{j=1}^{k} c \sqrt{x_{i}(t) x_{j}(t)} d b_{i j}(t) .
$$

The diffusion process governed by equation (18) is expressed as,

$$
d x_{i}(t)=M_{i} d t+\sum_{j=1}^{k} \sqrt{x_{i}(t) x_{j}(t)} d b_{i j}(t) .
$$


Thus the corresponding Fokker-Planck equation of Eq.(28) is a special case of the equation (18), putting $\varepsilon_{i}=1 / 2$ for all $i$ and taking $c=1$.

The transition probability density $p\left(\mathbf{x}, t \mid \mathbf{x}^{\prime}, 0\right)$ for equation (18) with $\varepsilon_{i}=1 / 2$, $i=, \ldots, k$ on the relative abundances $\mathbf{x}=\left(x_{1}, \ldots, x_{k}\right)$ starting from $\mathbf{x}^{\prime}=\left(x_{1}^{\prime} \ldots, x_{k}^{\prime}\right)$ is represented by using the transition probability density $\rho\left(\mathbf{y}, t \mid \mathbf{y}^{\prime}, 0\right)$, for equation (16)), on $\mathbf{y}=\left(y_{1}, \ldots, y_{k}\right)$ with spherical coordinates $\theta_{1}, \ldots, \theta_{k}$ of $(k-1)$-dimensional sphere starting from $\mathbf{y}^{\prime}=\left(y_{1}^{\prime}, \ldots, y_{k}^{\prime}\right)$ with spherical coordinates $\theta_{1}^{\prime}, \ldots, \theta_{k}^{\prime}$, as

$$
\begin{aligned}
& p\left(\mathbf{x}, t \mid \mathbf{x}^{\prime}, 0\right)\left|\frac{\partial\left(x_{1}, \ldots, x_{k-1}\right)}{\partial\left(\theta_{1}, \ldots, \theta_{k-1}\right)}\right| d \theta_{1} \ldots d \theta_{k-1} \\
& =\sum_{y_{i}^{\prime 2}=x_{i}^{\prime}, 0 \leq y_{i}^{\prime}, y_{i}^{2}=x_{i}, i=1, \ldots, k} \rho\left(\mathbf{y}, t \mid \mathbf{y}^{\prime}, 0\right)\left|\frac{\partial\left(y_{1}, \ldots, y_{k-1}\right)}{\partial\left(\theta_{1}, \ldots, \theta_{k-1}\right)}\right| d \theta_{1} \ldots d \theta_{k-1} .
\end{aligned}
$$

We have

$$
\left|\frac{\partial\left(x_{1}, \ldots, x_{n-1}\right)}{\partial\left(\theta_{1}, \ldots, \theta_{n-1}\right)}\right|=2^{k-1}\left(\prod_{i=1}^{k} y_{i}\right)\left|\frac{\partial\left(y_{1}, \ldots, y_{k-1}\right)}{\partial\left(\theta_{1}, \ldots, \theta_{k-1}\right)}\right| .
$$

Considering equation (16), (??), (17), (32) we have

$$
\begin{aligned}
& p\left(\mathbf{x}, t \mid \mathbf{x}^{\prime}, 0\right) \\
= & \frac{\Gamma(k / 2)}{\pi^{k / 2}}\left(\prod_{i=1}^{k} x_{i}^{-1 / 2}\right) 2^{-k} \sum_{y_{i}^{\prime 2}=x_{i}^{\prime}, 0 \leq y_{i}^{\prime}, y_{i}^{2}=x_{i}, i=1, \ldots, k} \\
& \sum_{L=0}^{\infty} \frac{2 L+k-2}{k-2} C_{L}^{k / 2-1}\left(\mathbf{y} \cdot \mathbf{y}^{\prime}\right) \exp (-D L(L+k-2) t) .
\end{aligned}
$$

Thus we proved the solution (33) is equivalent with the solution (20) for the case $\varepsilon=1 / 2$. Let us observe the two forms of the solution. Consider the eigen values of the two diffusion equations (9) and (18). The Caillol solution [3] of (9), expanded by the Gegenbauer polynomials, are applied in equation (33). The Gegenbauer polynomials for odd $L$ in equation (33) cancel with each other as we can see from equation (17). Hence there is no term for odd $L$ in equation (33), [17]. Put $L=2 n$, and take $D=1 / 8(c=1$ in (7)), for particular mutation rate $\varepsilon_{i}=1 / 2, \quad i=1, \ldots, k$. Then the exponential functions $\exp (-D L(L+k-2) t)$ in equation (33) coincide with those of Griffiths solution (20). The above $Q_{0}\left(\mathbf{x}, \mathbf{x}^{\prime}\right), Q_{1}\left(\mathbf{x}, \mathbf{x}^{\prime}\right)$, and $Q_{2}\left(\mathbf{x}, \mathbf{x}^{\prime}\right)$ in the solution (20) are obtained from the terms in the expansion (33) by using $x_{i}=y_{i}^{2}$ and $x_{i}^{\prime}=y_{i}^{\prime 2}$ for $i=1, \ldots, k$, as we can calculate easily. Also we see

$$
\frac{\Gamma(k / 2)}{\pi^{k / 2}}\left(\prod_{i=1}^{k} x_{i}^{-1 / 2}\right)=\Gamma(\mu) \prod_{i=1}^{k} \frac{x_{i}^{\varepsilon-1}}{\Gamma(\varepsilon)} .
$$

Our study naturally connects the diffusion on a hypersphere with the Wright-Fisher model for a particular mutation rate of the parent-independent mutation.

Remark 1. The connection between diffusion on a hypersphere and the 1dimensional Wright-Fisher diffusion has been derived before (p338 in [15] ). The equation (13.33) in [15] corresponds to equation (16). They derive the spectral expansion 
for a wider range of parameters. To extend this argument to the multidimensional case is one of our next problems.

Remark 2. The eigenfunctions in our equation (33) and the eigen function (21) in the Griffiths solution for the particular mutation rate look different, although they are identical. We do not have the algebraic proof in our present work and wish to have it in our next work. The form of the polynomials in our expansion means that they are the reproducing kernel polynomials for orthogonal polynomials on the Dirichlet measure [10], because all pairs of polynomials of the same degree are added to form the coefficient of a given eigenvalue.

Acknowledgement. The authors thank Hidenori Tachida for the references in population genetics. The authors thank Shinichi Kotani, Tohru Ogawa and Geoffrey A. Watterson for helpful discussion and suggestion on the symmetry of special functions. The authors thank the reviewers for helpful comments and suggestions.

[1] Brillinger, D. R. (1997). A Particle Migrating Randomly on a Sphere. Journal of Theoretical Probability, 10(2), 429-443.

[2] Carlsson, T., Ekholm, T., \& Elvingson, C. (2010). Algorithm for generating a Brownian motion on a sphere. Journal of physics A: Mathematical and theoretical, 43(50), 505001.

[3] Caillol, J. M. (2004). Random walks on hyperspheres of arbitrary dimensions. Journal of Physics A: Mathematical and General, 37(9), 3077.

[4] Debye P. (1929) Polar molecules, Dover, London.

[5] Ewens, W. J. (2004) Mathematical Population Genetics 1: I. Theoretical Introduction.Springer Science \& Business Media, Vol. 27. .

[6] Fisher, R. A. (1922) On the dominance ratio, Proceedings of the Royal Society of Edinburgh, 42, 321-341.

[7] Griffiths, R. C. (1979). A transition density expansion for a multi-allele diffusion model. Advances in Applied Probability, 310-325.

[8] Griffiths, R. C. (1980). Allele frequencies in multidimensional Wright-Fisher models with a general symmetric mutation structure. Theoretical Population Biology, 17(1), 51-70.

[9] Griffiths, B. (2009). Stochastic processes with orthogonal polynomial eigenfunctions. Journal of computational and applied mathematics, 233(3), 739-744.

[10] Griffiths, R. C., and Spano, D. (2013). Orthogonal polynomial kernels and canonical correlations for Dirichlet measures. Bernoulli, 19(2), 548-598.

[11] Itoh, Y. (1979). Random collision process on oriented graph. Institute of Statistical Mathematics (Japan), Research Memorandum, (154), 1-20.

[12] Itoh, Y. (1979). Random collision models in oriented graphs. Journal of Applied Probability, 36-44.

[13] Itoh, Y. (1993). Stochastic model of an integrable nonlinear system. Journal of the Physical Society of Japan, 62(6), 1826-1828.

[14] Itoh, Y., Mallows, C., and Shepp, L. (1998) Explicit sufficient invariants for an interacting particle system." Journal of Applied Probability, 35, 633-641.

[15] Karlin, S. and Taylor, H. (1981) A second course in stochastic processes, Academic Press, New York.

[16] Kimura, M. (1956). Random genetic drift in a tri-allelic locus; exact solution with a continuous model. Biometrics, 12(1), 57-66.

[17] Maruyama, K. (1992) Generalized Random Frequency Modulation Model and Its Applications, PhD Dissertation, The Graduate University for Advanced Studies (in Japanese).

[18] Maruyama, K. and Itoh, Y. (1991) Stochastic differential equations for the Wright-Fisher model, Proceedings of the Institute of Statistical Mathematics, 39, 47-52 (in Japnese with English sumary).

[19] Maruyama, T., and Nei, M. (1981). Genetic variability maintained by mutation and overdominant 
selection in finite populations. Genetics, 98(2), 441-459.

[20] McKean, H. P. (1969). Stochastic integrals (Vol. 353). American Mathematical Soc..

[21] Moran, P. A. P. (1958) Random processes in genetics, Mathematical Proceedings of the Cambridge Philosophical Society. Vol. 54. No. 01. Cambridge University Press, 1958.

[22] Perrin, M. F. (1928). Mouvement brownien de rotation. Ann. I'Ecole Norm. Sup. 45, 1-51.

[23] Takahata, N. (1981) Genetic variability and rate of gene substitution in a finite population under mutation and fluctuating selection. Genetics 98, 427-440.

[24] Vilenkin, N. I. (1978). Special functions and the theory of group representations (Vol. 22). American Mathematical Soc..

[25] Watterson, G.A. (1976) The stationary distribution of the infinitely-many neutral alleles diffusion model. J. Appl. Prob. 13, 639-651.

[26] Wright, S. (1931). Evolution in Mendelian populations. Genetics, 16(2), 97.

[27] Wright, S. (1969) Evolution and the genetics of populations. Vol.2 University of Chicago Press, Chicago.

[28] Yosida, K. (1949) Brownian motion on the surface of the 3-sphere." The Annals of Mathematical Statistics, 292-296. 